\title{
NLStradamus: a simple Hidden Markov Model for nuclear localization signal prediction
}

\author{
Alex N Nguyen $\mathrm{Ba}^{1,2}$, Anastassia Pogoutse ${ }^{1}$, Nicholas Provart ${ }^{1,2}$ and \\ Alan M Moses*1,2
}

\begin{abstract}
Address: ${ }^{1}$ Department of Cell \& Systems Biology, University of Toronto, 25 Willcocks Street, Toronto, Canada and ${ }^{2}$ Centre for the Analysis of Genome Evolution and Function, University of Toronto, 25 Willcocks Street, Toronto, Canada
\end{abstract}

Email: Alex N Nguyen Ba - alex.nguyenba@utoronto.ca; Anastassia Pogoutse - a.pogoutse@utoronto.ca; Nicholas Provart - nicholas.provart@utoronto.ca; Alan M Moses* - alan.moses@utoronto.ca

* Corresponding author

Published: 29 June 2009

BMC Bioinformatics 2009, 10:202 doi:10.1186/147/-2105-10-202
Received: 25 March 2009

Accepted: 29 June 2009

This article is available from: http://www.biomedcentral.com//47/-2/05//0/202

(c) 2009 Nguyen Ba et al; licensee BioMed Central Ltd.

This is an Open Access article distributed under the terms of the Creative Commons Attribution License (http://creativecommons.org/licenses/by/2.0), which permits unrestricted use, distribution, and reproduction in any medium, provided the original work is properly cited.

\begin{abstract}
Background: Nuclear localization signals (NLSs) are stretches of residues within a protein that are important for the regulated nuclear import of the protein. Of the many import pathways that exist in yeast, the best characterized is termed the 'classical' NLS pathway. The classical NLS contains specific patterns of basic residues and computational methods have been designed to predict the location of these motifs on proteins. The consensus sequences, or patterns, for the other import pathways are less well-understood.

Results: In this paper, we present an analysis of characterized NLSs in yeast, and find, despite the large number of nuclear import pathways, that NLSs seem to show similar patterns of amino acid residues. We test current prediction methods and observe a low true positive rate. We therefore suggest an approach using hidden Markov models (HMMs) to predict novel NLSs in proteins. We show that our method is able to consistently find $37 \%$ of the NLSs with a low false positive rate and that our method retains its true positive rate outside of the yeast data set used for the training parameters.
\end{abstract}

Conclusion: Our implementation of this model, NLStradamus, is made available at: http:// www.moseslab.csb.utoronto.ca/NLStradamus/

\section{Background}

Eukaryotic cells are defined by the presence of their nucleus. The nuclear membrane enclosing the genetic material of the cell is selective in its import of material through its nuclear pores and this translocation is mediated by cellular mechanisms [1,2].

Proteins entering the nucleus must do so through proteins forming the nuclear pores: the nuclear pore complex $[3,4]$. The pores allow the passive diffusion of small pro- teins, but bigger proteins entering the nucleus are usually bound by karyopherin complexes on their nuclear localization signal [5]. Although there are many nuclear import pathways in eukaryotic cells, most of these have not been characterized in detail. The best understood is the classical NLS pathway. The recognition of classical NLSs on nuclear proteins is done by the importin- $\alpha$ subunit which in turn is recognized by the importin- $\beta$ subunit. This trimer (cargo, importin- $\alpha$ and importin- $\beta$ ) is then imported to the nucleus after series of enzymatic steps $[1,6]$. Other 
families of NLSs are independent of importin- $\alpha$, and may bind directly to one of the members of the importin- $\beta$ superfamily [1].

Classical NLSs show characteristic patterns of basic residues loosely matching two consensus sequences, $\mathrm{K}(\mathrm{K} /$ $\mathrm{R}) \mathrm{X}(\mathrm{K} / \mathrm{R})$ and $\mathrm{KRX}_{10-12} \mathrm{KRXK}$, termed the 'monopartite' and 'bipartite' classical NLS [1,2]. PSORT [7] accurately predicts protein localization by including heuristic scores based on residues frequencies, protein domains as well as motifs. PSORT will then offer, based on the cNLS consensus sequences, various possible NLSs on nuclear proteins.

However, many known NLSs do not match any of the consensus sequences described above. Therefore, other computational methods have been proposed to predict NLSs based on amino acid sequences. One method, PredictNLS [8], employs a database of regular expressions to predict the various types of NLSs.

Here we sought to test the power of these methods to predict NLSs in Saccharomyces cerevisiae. We compiled a list of experimentally verified NLSs in yeast and found that PredictNLS [8] predicts $10 \%$ of the NLSs in this set. Based on an analysis of the residues frequencies in the NLSs in this set, we developed a simple hidden Markov model [9] that can be used to predict the nuclear localization signal in proteins, achieving true positive rate of $37 \%$. We also explored using specific models for the monopartite and bipartite NLSs, but surprisingly, found no significant improvement in positive predictive value. Finally, we also show that our method fares well in species other than yeast, consistent with the conservation of import mechanisms.

\section{Results \\ A set of experimentally verified nuclear localization signals in yeast}

In order to test the predictive power of computational methods to predict NLSs in S. cerevisiae, we sought to identify a set of experimentally confirmed NLSs. We searched the literature for papers in which specific stretches of amino acids have been shown to act as functional NLSs in yeast. While many approaches have been used to characterize and identify nuclear localization signals in proteins, we took as the NLS the minimal sequence in the protein that was proven to be either necessary or sufficient for nuclear import.

To categorize NLSs into their respective import pathways, we looked for evidence of their respective pathways which includes receptor binding or pathway dependency. However, only half of our proteins had a clear reference to these, and many remain unknown.
In all, we found 60 NLSs. Of these, 16 had evidence of importin- $\alpha$ dependence or binding and we here refer to these as cNLSs. In addition, we found 15 NLSs which depend on other import pathways, which we term noncNLSs. Finally, for 29 of our sequences, we failed to find information about the mechanism of import. The NLSs and references are listed in Tables 1 and 2.

\section{Current NLS prediction methods show little predictive power on our yeast data}

In order to test the power of NLS prediction methods, we first used the consensus sequence-based approach PredictNLS [8] and found that it was generally too specific: only $10 \%(6 / 60)$ of our characterized NLSs were identified by their genome wide analysis of the yeast proteome (see Figure 1a). These results were surprising, considering that this method had been reported to find $100 \%$ of the experimentally characterized NLSs considered in the general study [8]. We suggest that PredictNLS might be underestimating the true variability of NLSs, as few yeast NLSs (9) were included in that study. PredictNLS also produced 6 predictions that were not characterized NLSs, suggesting a positive predictive value of $50 \%$.

Probabilistic models have been widely used to predict domains in proteins (E.g., Pfam [10]). Profile HMMs model a linear series of states which approximately corresponds to the pattern of residues in a consensus motif. These models can account for length variation using insertion and deletion states.

NLSs with different import mechanisms are unlikely to share a consensus motif. Therefore, we tried a profile HMM approach to predict only cNLSs. We manually aligned the labelled cNLSs using a proposed biological model of binding specificity of importin- $\alpha$ [9] (Figure 2) and built an HMM model using HMMbuild [11] (see Methods). We used this model to predict cNLSs using HMMsearch, and assessed the predictive power at different E-value thresholds using a leave-one-out cross-validation (see Methods). The results are displayed as ROC curves in Figure 1b (see Methods) and indicate that HMMer obtains a similar positive predictive value (PPV) as PredictNLS at varying true positive rate (TPR).

Taken together, our results indicate that both frameworks show some positive predictive value but PredictNLS shows a low true positive rate, and both methods show PPV of around $50 \%$ in our data.

\section{The nuclear localization signal shows a strong statistical difference in residue frequencies}

While the NLSs can be divided in many functional categories depending on their pathways, we observed that most NLSs had an enrichment of basic residues. We first ana- 
Table I: non-cNLS and cNLS sets

\begin{tabular}{|c|c|c|c|c|c|}
\hline ORF & Gene name & Start & Stop & Necessity or Sufficiency (PMID) & Receptor binding or pathway dependency (PMID) \\
\hline YBR009C & HHFI & 4 & 21 & 11694505 & $11694505^{b}$ \\
\hline YBROIOW & HHTI & 10 & 28 & 11694505 & $11694505^{b}$ \\
\hline YDL007W & RPT2 & II & 15 & 15210724 & $15210724^{a}$ \\
\hline YDL007W & RPT2 & 33 & 37 & 15210724 & $15210724^{a}$ \\
\hline YDRI03W & STE5 & 49 & 66 & 10481914 & $10481914 b$ \\
\hline YDRI46C & SWI5 & 636 & 655 & $7615496 / 1652372$ & $18485366^{a}$ \\
\hline YDR208W & MSS4 & 347 & 364 & 12912920 & $12912920^{b}$ \\
\hline YDR224C & HTBI & 30 & 36 & 3123916 & I5679097b \\
\hline YEL009C & GCN4 & 231 & 246 & 12455686 & $18485366^{a}$ \\
\hline YER040W & GLN3 & 388 & 394 & 12624103 & $12624 \mid 03 / 18485366^{a}$ \\
\hline YFR034C & $\mathrm{PHO} 4$ & $14 \mid$ & 166 & 9732266 & $9732266^{b}$ \\
\hline YGL07IW & AFTI & 202 & 207 & 14523005 & $14523005^{b}$ \\
\hline YGL07IW & AFTI & 352 & 355 & 14523005 & $14523005^{b}$ \\
\hline YGL07IW & AFTI & 332 & 335 & 14523005 & $14523005^{b}$ \\
\hline YGL097W & SRMI & 3 & 23 & 18485366 & $18485366^{a}$ \\
\hline YHR079C & IREI & 645 & 657 & 17035634 & $17035634^{a}$ \\
\hline YIL075C & RPN2 & 811 & 832 & 15210724 & $15210724^{a}$ \\
\hline YILI50C & MCMIO & 512 & 527 & 13680157 & $18984568^{a}$ \\
\hline YILI50C & MCMIO & 435 & 451 & $13680 \mid 57$ & $18984568^{a}$ \\
\hline YJLI94W & $\mathrm{CDC} 6$ & 27 & 33 & 18485366 & $18485366^{a}$ \\
\hline YLRI03C & CDC45 & 209 & 228 & 18485366 & $18485366 / 18984568^{a}$ \\
\hline YLRI82W & SWI6 & 157 & 169 & 14998990 & $14998990^{a}$ \\
\hline YML007W & YAPI & 5 & 59 & || $274|4|$ & || $274|4|^{b}$ \\
\hline YMRI27C & SAS2 & 19 & 35 & 15788653 & I5788653b \\
\hline YMR239C & RNTI & 461 & 466 & 15090619 & $15337846^{a}$ \\
\hline YNL027W & CRZI & 394 & 422 & 11535618 & $11535618^{b}$ \\
\hline YNL027W & CRZI & 612 & 615 & 11535618 & $11535618^{b}$ \\
\hline YOLI23W & HRPI & 522 & 534 & 18343812 & $18343812^{b}$ \\
\hline YOLI27W & RPL25 & 18 & 28 & 1920406 & $9182759 / 9687515^{b}$ \\
\hline YOLI27W & RPL25 & 11 & 17 & 1920406 & $9182759 / 9687515^{b}$ \\
\hline YPLI53C & RAD53 & 785 & 807 & 15972895 & $15972895^{a}$ \\
\hline YPRII9W & CLB2 & 183 & 200 & 18485366 & $18485366^{a}$ \\
\hline
\end{tabular}

Characterized yeast nuclear localization signals used in our analysis for which import pathway is known. a corresponds to classical NLSs, b corresponds NLSs known not to dependent on the classical NLS import pathway. See text for details of how NLSs were classified. Numbers in the last two columns indicate references using pubmed IDs (PMID).

lyzed the residue content of the characterized cNLSs. Consistent with the definition of classical NLSs, we observe significant frequency differences (see Methods) mainly in lysine $\mathbf{7 . 3 \%}$ for the genome vs $29.3 \%$ for the cNLSs, Pvalue $\left.<10^{-10}\right)$ and arginine $(4.4 \%$ for the genome vs $15.7 \%$ for the cNLSs, P-value $<10^{-10}$ ).

To determine whether there were differences between the different types of NLSs, We then compared the set of cNLS to all other NLS (non-cNLS and unknown) and surprisingly found no significant differences in their residue frequencies. Thinking that maybe the unknown set of NLSs might have been mostly composed of cNLSs, we also compared the cNLS residue frequencies to the non-cNLS residue frequencies and also found no significant differences in residue frequencies. Thus, in our data set the three categories of NLSs (cNLS, non-cNLS and unknown) show no difference in residue frequencies.
Because the most apparent statistical difference lies in the frequencies of lysine (K) and arginine (R), we plotted each NLS in respect to number of $K$ and $R$ residues and their length (Figure 3), and consistent with the previous residue frequencies test, we found that there were generally no discernable differences between the three categories of NLSs.

Interestingly, we also observe two groups of NLSs on this plot, the first showing an average length of 8 amino acids containing an average of 3.9 basic residues while the other showing an average length of 20 amino acids containing an average of 7.3 basic residues. We suggest that these regions correspond to monopartite and bipartite NLSs [1]. We note that even NLSs known not to be importin- $\alpha$ dependent showed this pattern.

\section{A simple Hidden Markov Model shows better predictive performance}

Motivated by the idea that all NLSs in our set shared a similar bias in lysines and arginines, we created a simple two- 
Table 2: unknown NLS set

\begin{tabular}{|c|c|c|c|c|}
\hline ORF & Gene name & Start & Stop & Necessity or Sufficiency (PMID) \\
\hline YAL040C & CLN3 & 559 & 580 & II50967| \\
\hline YBLI05C & $\mathrm{PKCl}$ & 810 & 813 & 15643058 \\
\hline YBR098W & MMS4 & 244 & 263 & |464257| \\
\hline YCLOI7C & NFSI & 312 & 316 & 11110795 \\
\hline YCL067C & HMLALPHA2 & 1 & 13 & 1976249 \\
\hline YCL067C & HMLALPHA2 & $|4|$ & 159 & 1976249 \\
\hline YCR039C & MATALPHA2 & 2 & 13 & 8757785 \\
\hline YCR039C & MATALPHA2 & $|4|$ & 159 & 8757785 \\
\hline YDR034C & LYSI4 & 190 & 250 & 10975256 \\
\hline YEL032W & MCM3 & 766 & 772 & 16093348 \\
\hline YEL06IC & CIN8 & 994 & 1000 & 11694576 \\
\hline YGLI03W & RPL28 & 24 & 30 & 2104804 \\
\hline YGLI03W & RPL28 & 7 & 14 & 2104804 \\
\hline YGR027C & RPS25A & 11 & 36 & 10386617 \\
\hline YGR027C & RPS25A & 87 & 95 & 10386617 \\
\hline YIR006C & PANI & 1024 & 1040 & 17967424 \\
\hline YIR006C & PANI & 1145 & 1161 & 17967424 \\
\hline YJLI57C & FARI & 11 & 30 & 10485850 \\
\hline YJLI57C & FARI & 38 & 48 & 10485850 \\
\hline YJLI87C & SWEI & 304 & 310 & 18562688 \\
\hline YJLI90C & RPS22A & 21 & 29 & 10386617 \\
\hline YKLIII2W & $A B F I$ & 624 & 628 & 15522095 \\
\hline YLR079W & $\mathrm{SICl}$ & 77 & 89 & 16294029 \\
\hline YML024W & RPSI7A & 2 & 7 & 3939318 \\
\hline YMR036C & $\mathrm{MIHI}$ & 31 & 33 & 18562688 \\
\hline YOR063W & RPL3 & I & 21 & 3931077 \\
\hline YOR274W & MOD5 & 408 & 424 & 9872948 \\
\hline YPRI89W & SKI3 & 306 & 314 & 2660461 \\
\hline
\end{tabular}

Characterized yeast nuclear localization signals used in our analysis, for which we could not find evidence for a specific import pathway. Numbers in the last column are pubmed IDs for references. See text for details of how NLSs were classified.

state HMM. In this model, sequence is generated either from a background model (with residue frequencies equal to those in the genome) or an NLS model, whose residue frequencies are equal to those in all of the characterized yeast NLSs (Figure 4a). To assess the positive predictive value of the model, we performed a leave-one-out cross validation, as above (see Methods), of the HMM. We assessed its predictive strength in two ways, either using the most probable path (the Viterbi algorithm) or by computing the posterior probability and counting predictions when it passed a certain threshold (see Methods). Relative to PredictNLS, the results show significantly improved positive predictive value $(\mathrm{PPV}=88 \%$ vs. $\mathrm{PPV}=50 \%, \mathrm{P}-$ value $<0.05)$, and higher true positive rate $(\mathrm{TPR}=37 \%$ vs. $\mathrm{TPR}=10 \%$, P-value $<0.001$, Figure 5) while producing only 3 false positives. Our results were specific to the experimentally defined NLSs. We defined a residue-level correlation coefficient (analogous to nucleotide level correlation coefficient $[12,13]$ for DNA motifs) and found that our model yielded a coefficient of 0.36 . We also computed the Matthews Correlation Coefficient [14] and found that it was 0.55 , similar to recent results for a predictor of nuclear export signals [15].
We were surprised to observe that a single, simple model could achieve increased performance even though our training data spanned a large diversity of NLSs, e.g., some cNLSs and others not, both bipartite and monopartite. Nevertheless, we found that our method showed similar positive predictive value on each set of NLSs when we analyzed them individually (data not shown). While perhaps surprising, these results are consistent with the similar residue compositions of each sets of NLSs (Figure 3 ).

\section{A more complex model does not improve prediction}

We observed two main regions within our K-R content plot where NLSs seemed to aggregate and this is consistent for the bipartite and monopartite classes of cNLSs, but we were surprised to see that this was also observed in the other sets. To test whether or not we could improve our predictions using this information, we created a four state HMM by modelling a 'spacer' state between two patches of basic residues (Figure $4 \mathrm{~b}$, see methods) and analyzed its predictive power using a leave-one-out cross-validation. This model is able to recognize the two basic stretches of the NLS separated by a spacer region, which we model using the background residue frequencies. We refer to this model as the 'bipartite model'. In some cases, this model accurately identifies the boundaries 


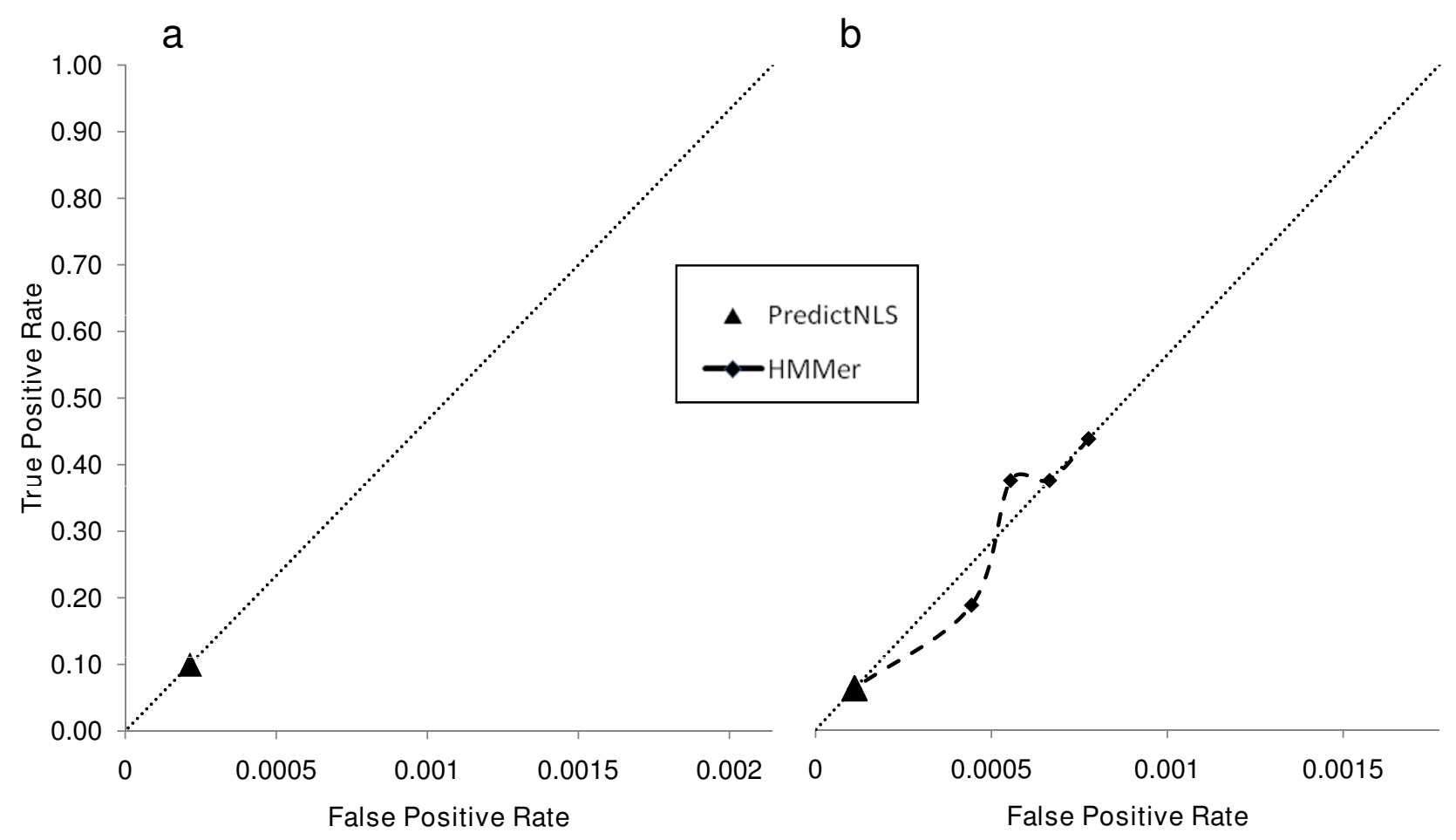

Figure I

True positive and false positive rate of consensus and alignments based methods. a) True positive and false positive rate of a consensus-based method on all NLSs from our dataset. The false positive rate is shown as the error rate per amino acid residue. The diagonal line depicts a ratio of one true prediction per false prediction per amino acid residue. b) True positive and false positive rate of consensus and alignment based methods on classical NLSs from our dataset. The false positive rate is shown as the error rate per amino acid residue. The diagonal line depicts a ratio of one true prediction per false prediction per amino acid residue.

of these regions (Figure 6a, c). For comparison, we also trained a model using only the shorter NLSs, which we refer to as the 'monopartite model'. We refer to the initial model that uses the frequency of all of the NLSs as the 'combined model'.

To test these models, we defined bipartite and monopartite NLSs based on the two classes we had observed in Figure 3. We tested the bipartite model on the bipartite NLSs only. Surprisingly, the positive predictive value and true positive rate of the bipartite model was exactly the same as the combined model (TPR $=61 \%$, same FPR), as the combined model can also identify bipartite NLSs (Figure 6b, d). Similar predictive power was also observed when comparing the monopartite model to the combined model on only monopartite NLSs. However, a small increase in true positive rate was observed for the monopartite model ( $\mathrm{TPR}=41 \%$ vs 38\%, same FPR) but this difference was not significant.

\section{Applying the model to other organisms}

We were concerned that our predictor might have a bias for yeast proteins. We therefore tested our method on the set of NLSs used to train PredictNLS [8]. This set of data contains NLSs that have been shown to function in vertebrate cells. We found that our method has a similar true positive rate in this data as it does in yeast, finding $37 \%$ of the characterized NLSs at the same posterior threshold. However we do note an increased false positive rate $(228 \%$ increase, $\mathrm{P}$-value $=0.08)$, and modest reductions in the nucleotide-level correlation coefficient to 0.30 (from 0.36, Figure 7) and the Matthews Correlation Coefficient to 0.48 (from 0.55 ).

Seeking to understand the elevated rate of false predictions, we explored them further and found that 7 of the 15 vertebrate false positives occur on only three proteins. When we searched the literature for more information on these proteins we found that one of our 'false' predictions had actually been identified as an NLS [16,17]. We identified on another protein two patches of amino-acid repeats, and finally on the last protein two nucleolar localization signals, which are long stretches of basic amino acid residues, and it is not yet clear if these signals should be considered as NLSs $[18,19]$. 


\begin{tabular}{|c|c|c|c|c|c|c|c|c|c|c|c|c|}
\hline & & \multicolumn{4}{|c|}{ Minor cNLS binding site } & \multirow[t]{2}{*}{ Spacer } & \multicolumn{5}{|c|}{ Major cNLS binding site } & \\
\hline & & $\mathrm{P} 1$ & $\mathrm{P} 2$ & P3 & P4 & & $\mathrm{P} 1$ & P2 & P3 & $\mathrm{P} 4$ & P5 & \\
\hline YLR103C CDC45 & & $\mathrm{K}$ & $\mathrm{R}$ & G & $\mathrm{N}$ & SSIGPNDLS & $\mathrm{K}$ & $\mathrm{R}$ & $\mathrm{K}$ & $\mathrm{Q}$ & $\mathrm{R}$ & KK \\
\hline YPR119W CLB2 & $\mathrm{K}$ & $\mathrm{K}$ & $\mathrm{R}$ & $\mathrm{P}$ & 1 & STIVEQEL & $\mathrm{P}$ & K & $\mathrm{K}$ & $\mathrm{F}$ & $\mathrm{K}$ & \\
\hline YDR146C SWI5 & & $\mathrm{K}$ & $\mathrm{K}$ & $\mathrm{Y}$ & $E$ & NVVIKRSP & $\mathrm{R}$ & K & $\mathrm{R}$ & $\mathrm{G}$ & $\mathrm{R}$ & PRK \\
\hline YGL097W PRP20 & & $\mathrm{K}$ & $\mathrm{R}$ & $\mathrm{T}$ & $\mathrm{V}$ & ATNGDASGAHRA & $\mathrm{K}$ & $\mathrm{K}$ & $\mathrm{M}$ & $\mathrm{S}$ & $\mathrm{K}$ & \\
\hline YEL009C GCN4 & & $\mathrm{K}$ & $\mathrm{R}$ & A & $\mathrm{R}$ & NTEAARRS & - & $\mathrm{R}$ & A & $\mathrm{R}$ & $\mathrm{K}$ & \\
\hline YIL075C RPN2 & KARA & $\mathrm{K}$ & $\mathrm{K}$ & $\mathrm{T}$ & $\mathrm{K}$ & KEKGPNEEE & - & $\mathrm{K}$ & $\mathrm{K}$ & $\mathrm{K}$ & $E$ & \\
\hline YIL150C MCM10 & & $\mathrm{K}$ & $\mathrm{K}$ & $\mathrm{S}$ & $\mathrm{T}$ & ALSRELG & - & K & 1 & $\mathrm{M}$ & $\mathrm{R}$ & $\mathrm{RR}$ \\
\hline YIL150C MCM10 & & $\mathrm{K}$ & $\mathrm{K}$ & $\mathrm{K}$ & $\mathrm{T}$ & VINDLLHY & - & $\mathrm{K}$ & $\mathrm{K}$ & $E$ & $\mathrm{~K}$ & \\
\hline YPL153C RAD53 & & $\mathrm{K}$ & $\mathrm{R}$ & 1 & $\mathrm{H}$ & SVSLSQSQIDP & $S$ & $\mathrm{~K}$ & $\mathrm{~K}$ & $\mathrm{~V}$ & $\mathrm{~K}$ & RAK \\
\hline YJL194W CDC6 & & & & & & $\mathrm{RP}$ & $\mathrm{L}$ & $\mathrm{K}$ & $\mathrm{R}$ & $\mathrm{K}$ & $\mathrm{K}$ & \\
\hline YER040W GLN3 & & & & & & $P$ & 1 & $\mathrm{R}$ & $S$ & $\mathrm{R}$ & $\mathrm{K}$ & K \\
\hline YHR079C IRE1 & & & & & & RKRGSRG & $\mathrm{G}$ & K & $\mathrm{K}$ & $\mathrm{G}$ & $\mathrm{R}$ & K \\
\hline YMR239C RNT1 & & & & & & K & $\mathrm{N}$ & $\mathrm{K}$ & $\mathrm{K}$ & $\mathrm{R}$ & $\mathrm{K}$ & \\
\hline YLR182W SWI6 & & & & & & ELGSP & $\mathrm{L}$ & K & $\mathrm{K}$ & L & $\mathrm{K}$ & IDT \\
\hline YDL007W RPT2 & & & & & & & $\mathrm{K}$ & $\mathrm{K}$ & $\mathrm{K}$ & $\mathrm{K}$ & $\mathrm{K}$ & \\
\hline YDL007W RPT2 & & & & & & & $\mathrm{R}$ & $\mathrm{K}$ & $\mathrm{K}$ & $\mathrm{R}$ & $\mathrm{K}$ & \\
\hline
\end{tabular}

\section{Figure 2}

Alignment of characterized classical nuclear localization signals. Alignment of the residues thought to contribute to NLS binding to importin- $\alpha$. The residues aligned on the cNLS major binding site were then used as model for a profile HMM approach using HMMer.

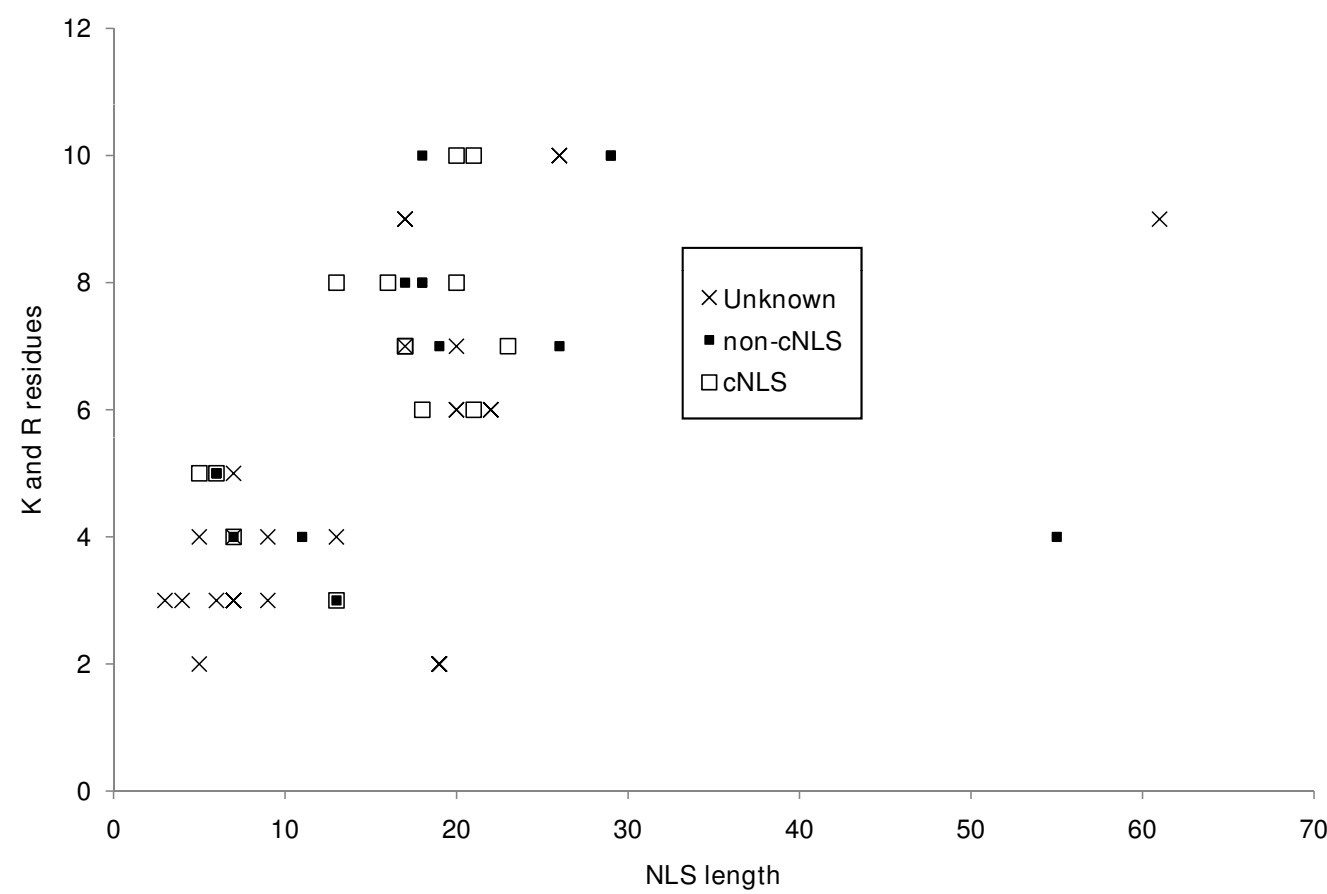

\section{Figure 3}

Lysine and arginine content of characterized nuclear localization signals. Plot of the lysine and arginine content of characterized nuclear localization signals with respect to their length. The plot shows the three 'types' of NLSs present in our study. 


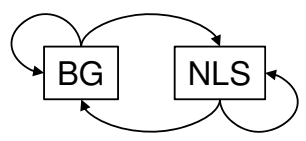

b

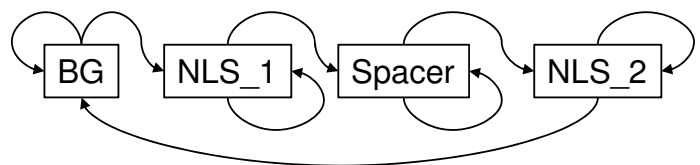

\section{Figure 4}

Schematic of our two state and four state HMM. a) The two state HMM models two states which are represented by the 'background', which emits residues with the same frequency as the genome, and by the 'NLS' state, which emits residues with the same frequency as the NLSs from our characterized data. b) The four state HMM models four states which are represented by the 'background', which emits residues with the same frequency as the genome, two 'NLS' states, which emit residues with the same frequency as our characterized NLSs, separated by a 'spacer' state which emits residues with the same frequency as the genome.

We noted in total three false predictions composed of amino-acid repeats, which were largely absent from our yeast training set. Such repeats are problematic because they violate an assumption of our simple hidden-Markov model, namely, that adjacent residues are independent given the value of the hidden state.

Taken together, this analysis of vertebrate NLSs demonstrates that the true positive rate of our method is not confined to our training data set. However, we did note an increase in false positives, which might be explained by additional undiscovered NLSs in this data set, or repetitive sequences in vertebrate proteins.

\section{Discussion}

Analysis of residue frequency clearly shows that there is a bias in key residues in the NLS, and while previous studies have shown that there exists some position requirement in these residues [20], the NLSs do not clearly align and therefore we believe that the signal simply do not obey a clear consensus sequence rule. Our results are consistent with the model that NLSs may simply be regions of high positive charges with only minor spatial requirements [20]. This idea is consistent with the model that the NLS can be masked by phosphorylation [21], by inhibiting the activity of the signal due to addition of negative charges.

There is abundant evidence that cNLSs bind at specific positions on importin- $\alpha$ and this binding is mediated by two sites which bind monopartite or bipartite cNLSs $[22,23]$, which suggests that the bipartite cNLS is not simply a bigger monopartite cNLS. We were, however, unable to exploit this knowledge to create a stronger predictor. While each basic patch in the bipartite cNLS does not represent a monopartite cNLS, the combination of both patches is sufficient for recognition by our predictor.

By examining some false negatives, we find at least two reasons why our method fails to predict them. First, there are examples that do not seem to show an enrichment of basic residues. Second, other NLSs are simply too small to be identified reliably, due to a lack of statistical signal.

We note that unlike PSORT which is based on the cNLS consensus motifs, our method does not attempt to predict NLSs belonging to a particular pathway. We observed that all NLSs show an excess of basic residues, and therefore we have developed a simple HMM that can identify stretches of basic residues in protein sequences. It is somewhat surprising that this method performs reasonably well. This suggests that the consensus patterns for different import pathways may be more similar than currently anticipated.

We believe that obtaining a higher positive predictive value is certainly possible by combining other biological

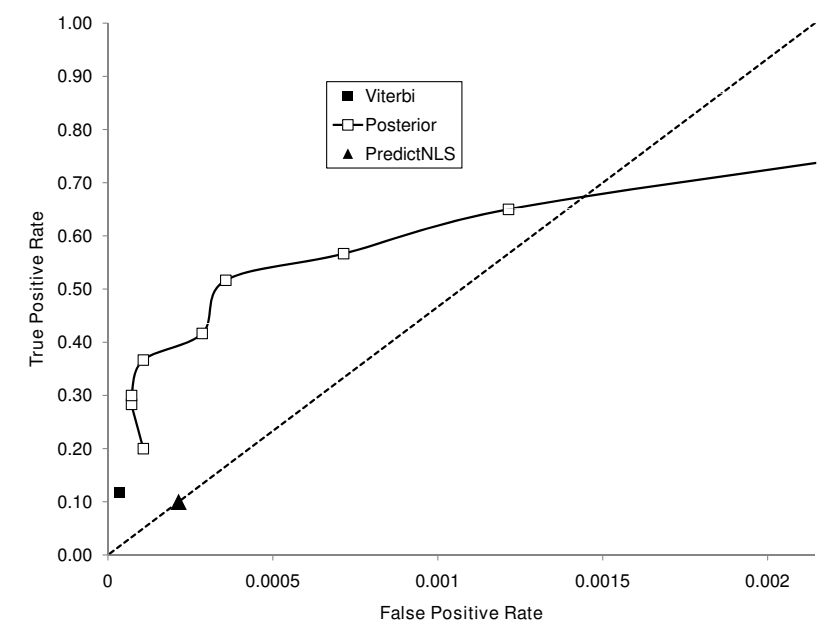

Figure 5

True positive and false positive rate of our model. True positive and false positive rate of various methods, including our HMM at various posterior threshold and the Viterbi algorithm on our dataset. The false positive rate is shown as the error rate per amino acid residue. The diagonal line depicts a ratio of one true prediction per false prediction per amino acid residue. 


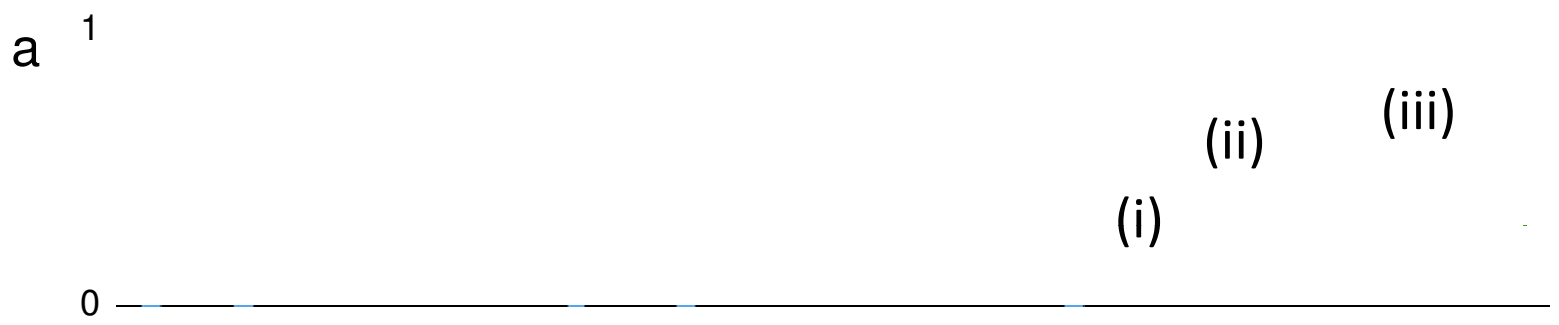

KKYENVVIKRSPRKRGRPRK

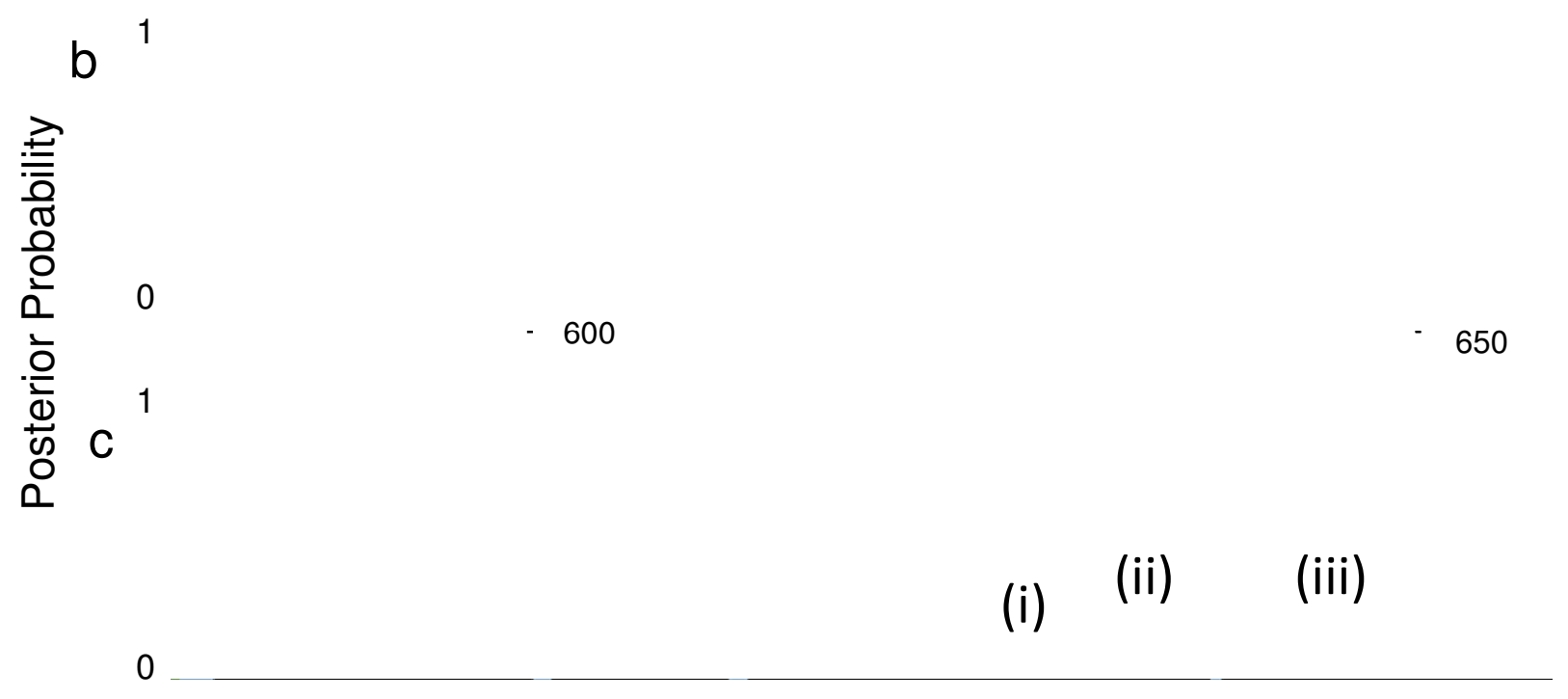

KKWTEKLARFQRSSAKKK

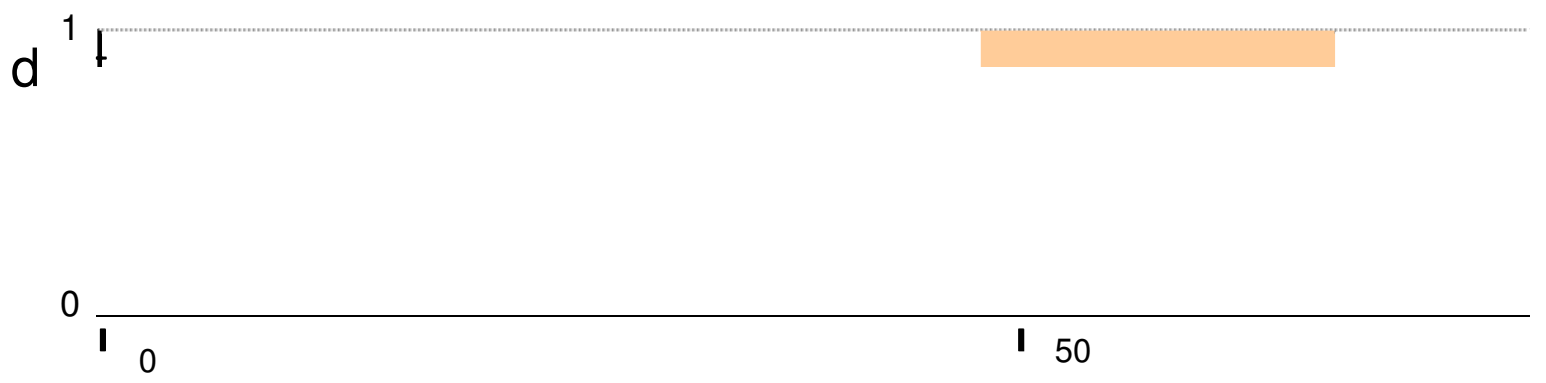

Position in protein (aa)

Figure 6

Posterior trace of Swi5p and Ste5p for our two HMMs. a) Posterior trace of Swi5p, a characterized bipartite cNLS, using our four state model. Output was generated by NLStradamus and highlighted region shows the region of characterized NLS. Black (i) and blue (iii) lines represent the two patches of basic residues while the pink line (ii) represents the spacer. Green line represents the sum of the three NLS states. Red line is shown as a reference for a threshold of 0.6 . b) Posterior trace of Swi5p, a characterized bipartite NLS, using our simple two state model. Output was generated by NLStradamus and highlighted region shows the region of characterized NLS. Horizontal red line depicts the chosen posterior threshold of 0.6 . c) Posterior trace of Ste5p, a characterized bipartite importin- $\beta$ dependent NLS (non-cNLS), using our four state model. Output was generated by NLStradamus and highlighted region shows the region of characterized NLS. Black (i) and blue (iii) lines represent the two patches of basic residues while the pink line (ii) represents the spacer. Green line represents the sum of the three NLS states. Red line is shown as a reference for a threshold of 0.6. d) Posterior trace of Ste5p, a characterized bipartite non-classical NLS, using our simple two state model. Output was generated by NLStradamus and highlighted region shows the region of characterized NLS. Horizontal red line depicts the chosen posterior threshold of 0.6. 


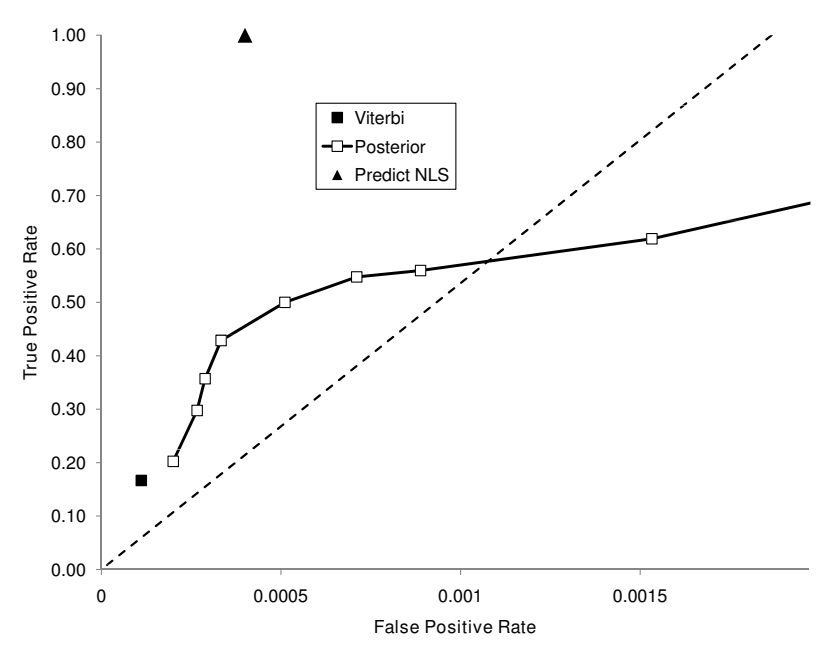

Figure 7

True positive and false positive rate of our model on other species. True positive and false positive rate of various methods, including our HMM at various posterior threshold and the Viterbi algorithm on the PredictNLS dataset. This ROC curve was created by counting overlaps. The false positive rate is shown as the error rate per amino acid residue. The diagonal line depicts a ratio of one true prediction per false prediction per amino acid residue.

knowledge; however, this is still under study. For example, some of our false predictions might either be real NLSs that have not yet been characterized, or simply part of other undiscovered protein domains.

\section{Conclusion}

In conclusion, we offer a simplified approach to predicting nuclear localization signals and show that this method can be applied to multiple species. This agrees with the notion that important regulatory mechanisms are conserved, although it is possible that differences in positive predictive value can be attributed to the existence of multiple importin- $\alpha, \beta$ homologues in other species [24] or differences in proteins lengths between species.

\section{Methods}

\section{Creation of alignments and assessment of consensus} sequence based methods

Based on recent studies on the binding sites of the importin- $\alpha$ [9], we created an alignment of our cNLS based on the major site of the importin (Figure 2). This alignment was run through HMMer [11] using HMMbuild and calibrated using HMMcalibrate. Using HMMsearch and a leave-one-out cross-validation [25], we assessed its predictive power. The leave-one-out cross-validation of the HMMer framework was done by removing all the characterized NLSs of one of the proteins from the alignment and then applying the above method on the protein.

\section{Analysis of residue frequency in the nuclear localization signal and statistical significance}

We first verified if the NLS containing proteins had different residue frequencies than the genome to verify if our proteins were different than expected in the genome and found that this was the case. We used a sampling analysis (data not shown) and a two-tailed Fisher's exact test. We computed

$$
P_{\text {cutoff }}=\frac{R_{1} ! R_{2} ! C_{1} ! C_{2} !}{N ! \prod a_{i j} !}
$$

where $a_{i j}$ is the number of counts in $i^{\text {th }}$ row and $j^{\text {th }}$ column of a $2 \times 2$ table, $R_{1}, R_{2}, C_{1}$ and $C_{2}$ are the row and column totals, respectively, and $N$ is the total number of observations. To calculate the two-tailed P-value using Fisher's exact test, we summed the probabilities of all the matrices below and equal to the $P_{\text {cutoff. }}$ If the P-value was below than 0.01 we considered that residue significant.

\section{Creation of a Hidden Markov model}

We created a simple two state HMM as well as a four state HMM to assess the difference between monopartite and bipartite motifs. The two state HMM receives two sets of states based on the background and NLS residue frequencies, while the four state HMM assumes that the spacer residue frequencies are equal to the 'background' residue frequencies. A four state HMM where the spacer residue frequencies were taken directly from our characterized data was also done (data not shown). Transition probabilities were approximated by summing the number of times that a transition occurs divided by the total amount of transitions from a given state in our data set. The starting state was assumed to be the same as the 'background' state. Further analysis using the Baum-Welch algorithm $[26,27]$ was used but did not yield significant predictive power difference. Outputs are both the most probable path and posterior probabilities. This HMM is publically accessible from our web page.

The transition probabilities between each state were calculated as:

$$
a_{k l}=\frac{A_{k l}}{\sum l^{\prime} A_{k l^{\prime}}}
$$

Where $\mathrm{a}_{\mathrm{kl}}$ is the transition probability from state $\mathrm{k}$ to state $\mathrm{l}$ and $\mathrm{A}_{\mathrm{kl}}$ is the observed frequency of this transition in the characterized data.

The posterior probability at a given position was calculated as in [26]:

$$
P\left(\pi_{i}=k \mid x\right)=\frac{f_{k}(i) b_{k}(i)}{P(x)}
$$


The posterior probability is the probability that the $\mathrm{ith}^{\text {th }}$ amino acid in the given sequence $(\mathrm{x})$ is produced by state $\mathrm{k}\left(\pi_{\mathrm{i}}=\mathrm{k}\right)$. This is calculated using the forward algorithm, which is the probability of all the sequences up to and including the $\mathrm{i}^{\text {th }}$ amino acid requiring that the $\mathrm{i}^{\text {th }}$ amino acid is produced by state $k\left(f_{k}(i)\right)$, multiplied by probability of all sequences after the $i^{\text {th }}$ amino acid when the $i^{\text {th }}$ amino acid is produced by state $\mathrm{k}\left(\mathrm{b}_{\mathrm{k}}(\mathrm{i})\right)$, given by the backward algorithm, and divided by the probability of the whole sequence given the model $(\mathrm{P}(\mathrm{x}))$.

\section{Signal detection, optimizing true positive rate and ROC curve}

We used several thresholds on the posterior probability and any segment over the threshold is labelled as a signal for the two state model. The four state model instead sums the two NLS states as well as the spacer state and establishes a signal when this sum is over the threshold. Using signal/true motif overlaps, we assess the true positive rate and positive predictive value using a leave-one-out crossvalidation [25]. The leave-one-out cross validation was done systematically by removing a protein from the training set, and testing the predictor on this protein.

The threshold whose ratio is furthest from a ratio of one true positive prediction per false prediction was deemed to be the strongest. This ratio, one true positive per false prediction, is shown in our ROC curves as a dotted diagonal line. We note that this diagonal is not indicative of a random predictor as in traditional ROC curves. We also calculated a residue correlation coefficient (rCC) [12] to assess performance of the model.

$$
r C C=\frac{T P^{*} T N-F P^{*} F N}{\sqrt{(T P+F P)(T P+F N)(T N+F P)(T N+F N)}}
$$

The rCC is the Pearson product-moment coefficient of correlation, where TP and TN are the number of true positives and true negatives, while FP and FN are the number of false positives and false negatives, taken at the nucleotide level as opposed to the signal level. Because our predictions are done on protein sequences, this was done at the residue level instead of nucleotides as is done for DNA motifs [12].

A Matthews' correlation coefficient is calculated similarly to the calculation of the $\mathrm{rCC}$, however this is taken at the signal level and taking as the number of true negatives the number of predictions made at a threshold of 0.001 . This is a similar method as used by predictNES $[14,15]$. This number of true negatives was also used to assess significance of false positive rate differences in a Fisher's test as above.

True positive rate (TPR) is calculated as the number of true predictions divided by the number of total possible true positives, while the false positive rate (FPR) is calculated as the number of false positives divided by the sum of the length of all our characterized proteins. The false positive rate is therefore given at the residue level, while the true positive rate is given at the level of individual NLSs. Positive predictive value (PPV) is assessed as the ratio of true positive predicted NLSs to the total number of predicted NLSs.

\section{Availability}

The HMM-based prediction method, NLStradamus, for predicting nuclear localization signals can be accessed directly at: http://www.moseslab.csb.utoronto.ca/ NLStradamus/. Both two state and four state HMMs are available for use and posterior decoding at different thresholds is also possible.

\section{Authors' contributions}

ANNB wrote the software, designed and performed the experiments, identified characterized motifs and wrote the paper. AP identified characterized motifs. NP provided insights on possible displays for the web software and edited the manuscript. AMM wrote the paper and designed the experiments. All authors have read and approved the final manuscript.

\section{Acknowledgements}

This work was supported by a research award from the Center for the Analysis of Genome Evolution and Function (CAGEF) to ANNB and by a Canadian Foundation for Innovation infrastructure grant to AMM. We thank members of NP's lab for providing us with valuable inputs during the beginning of the project, as well as providing us the web server, and Hardeep Nahal helped us set up our web application. We acknowledge members of CUBIC involved in the PredictNLS project, who kindly provided us a copy of their standalone program to help us do a prior analysis of the nuclear localization signal.

\section{References}

I. Lange A, Mills RE, Lange CJ, Stewart M, Devine SE, Corbett AH: J Biol Chem 2007, 282(8):5 I0I-5. Epub 2006 Dec 14.

2. Poon IK, Jans DA: Regulation of nuclear transport: central role in development and transformation? Traffic 2005, 6(3): I73-86.

3. Rout MP, Blobel G: Isolation of the yeast nuclear pore complex. J Cell Biol 1993, I23(4):771-83.

4. Panté N, Aebi U: The nuclear pore complex. J Cell Biol 1993, I 22(5):977-84.

5. Görlich D, Mattaj IW: Nucleocytoplasmic transport. Science |996, 27 I(5255): | 5 | 3-8.

6. Moroianu J, Blobel G, Radu A: Nuclear protein import: Ran-GTP dissociates the karyopherin alphabeta heterodimer by displacing alpha from an overlapping binding site on beta. Proc Natl Acad Sci USA 1996, 93( I 4):7059-62.

7. Nakai K, Horton P: PSORT: a program for detecting the sorting signals of proteins and predicting their subcellular localization. Trends Biochem Sci 1999, 24(I):34-35.

8. Cokol M, Nair R, Rost B: Finding nuclear localization signals. EMBO Rep 2000, I(5):4I I-5.

9. Hahn S, Maurer P, Caesar S, Schlenstedt G: Classical NLS proteins from Saccharomyces cerevisiae. J Mol Biol 2008, 379(4):678-94. Epub 2008 Apr 22.

10. Finn RD, Tate J, Mistry J, Coggill PC, Sammut JS, Hotz HR, Ceric G, Forslund K, Eddy SR, Sonnhammer EL, Bateman A: The PFAM protein familes database. Nucleic Acids Research 2008:D28I-D288. 
II. Eddy SR: Profile hidden Markov models. Bioinformatics 1998, I4(9):755-63.

12. Burset $M$, Guigó R: Evaluation of gene structure prediction programs. Genomics 1996, 34(3):353-67.

13. Tompa M, Li N, Bailey TL, Church GM, De Moor B, Eskin E, Favorov AV, Frith MC, Fu Y, Kent WJ, Makeev VJ, Mironov AA, Noble WS, Pavesi G, Pesole G, Régnier M, Simonis N, Sinha S, Thijs G, van Helden J, Vandenbogaert M, Weng Z, Workman C, Ye C, Zhu Z: Assessing computational tools for the discovery of transcription factor binding sites. Nat Biotechnol 2005, 23(I):137-44.

14. Matthews BW: Comparison of the predicted and observed secondary structure of T4 phage lysozyme. Biochim Biophys Acta 1975, 405(2):442-5I.

15. la Cour T, Kiemer L, Mølgaard A, Gupta R, Skriver K, Brunak S: Analysis and prediction of leucine-rich nuclear export signals. Protein Eng Des Sel 2004, 17(6):527-36. Epub 2004 Aug 16.

16. Kaneko H, Orii KO, Matsui E, Shimozawa N, Fukao T, Matsumoto T, Shimamoto A, Furuichi Y, Hayakawa S, Kasahara K, Kondo N: BLM (the causative gene of Bloom syndrome) protein translocation into the nucleus by a nuclear localization signal. Biochem Biophys Res Commun 1997, 240(2):348-53.

17. Mirski SEL, Gerlach JH, Cole SPC: Sequence Determinants of Nuclear Localization in the $\alpha$ and $\beta$ Isoforms of Human Topoisomerase II. Experimental Cell Research 1999, $251(2): 329-339$.

18. Dokal I: Dyskeratosis congenita in all its forms. $\mathrm{Br} J$ Haematol 2000, I I O(4):768-79.

19. Hatanaka M: Discovery of the nucleolar targeting signal. Bioessays 1990, I 2(3): 143-8.

20. Colledge WH, Richardson WD, Edge MD, Smith AE: Extensive mutagenesis of the nuclear location signal of simian virus $\mathbf{4 0}$ large-T antigen. Mol Cell Biol I986, 6( I I):4I36-9.

21. Jans DA, Hübner S: Regulation of protein transport to the nucleus: central role of phosphorylation. Physiol Rev 1996, 76(3):65I-85.

22. Conti E, Uy M, Leighton L, Blobel G, Kuriyan J: Crystallographic analysis of the recognition of a nuclear localization signal by the nuclear import factor karyopherin alpha. Cell 1998, 94(2): 1 93-204.

23. Fontes MR, Teh T, Kobe B: Structural basis of recognition of monopartite and bipartite nuclear localization sequences by mammalian importin-alpha. J Mol Biol 2000, 297(5): I I83-94.

24. Goldfarb DS, Corbett AH, Mason DA, Harreman MT, Adam SA: Importin alpha: a multipurpose nuclear-transport receptor. Trends Cell Biol 2004, I 4(9):505-I4.

25. Mosteller F, Tukey JW: Data analysis, including statistics. In Handbook of Social Psychology 2nd edition. Edited by: Lindzey G, Aronson E. Reading: Addison-Wesley; 1968:80-203.

26. Durbin R, Eddy S, Krogh A, Mitchison G: Biological sequence analysis: probabilistic models of proteins and nucleic acids Cambridge: Cambridge University Press; 1998.

27. Baum LE, Petrie T, Soules G, Weiss N: A Maximization Technique Occurring in the Statistical Analysis of Probabilistic Functions of Markov Chains. The Annals of Mathematical Statistics 1970, 4 I(I):164-17I.

Publish with Bio Med Central and every scientist can read your work free of charge

"BioMed Central will be the most significant development for disseminating the results of biomedical research in our lifetime. "

Sir Paul Nurse, Cancer Research UK

Your research papers will be:

- available free of charge to the entire biomedical community

- peer reviewed and published immediately upon acceptance

- cited in PubMed and archived on PubMed Central

- yours - you keep the copyright
BioMedcentral 